\title{
THE REDUCTION OF POTENTIAL DIFFUSIONS TO FINITE STATE MARKOV CHAINS AND STOCHASTIC RESONANCE
}

\author{
Peter Imkeller \\ Institut für Mathematik, Humboldt-Universität zu Berlin \\ Unter den Linden 6, 10099 Berlin, Germany \\ imkeller@mathematik.hu-berlin.de \\ Ilya Pavlyukevich \\ Institut für Mathematik, Technische Universität Berlin \\ Straße des 17. Juni 136, 10623 Berlin, Germany \\ pavljuke@math.tu-berlin.de
}

\begin{abstract}
Consider a dynamical system describing the motion of a particle in a double well potential with a periodic perturbation of very small frequency, and a white noise perturbation of intensity $\varepsilon$. If its trajectories amplify the small periodic perturbation in a 'best possible way', it is said to be in stochastic resonance. A lower bound for the ratio of amplitude and logarithm of the period above which quasi-deterministic periodic behavior can be observed is obtained via large deviations theory. However, to obtain optimality, periodicity of trajectories has to be studied by means of a measure of quality of tuning such as spectral power amplification. In the particular setting where the potential alternates every half period between two spatially antisymmetric double well states we encounter a surprise. The stochastic resonance pattern is not correctly described by the reduced dynamics associated with a two state Markov chain whose periodic hopping rates between the potential minima mimic the large (spatial) scale motion of the diffusion. Only if small scale fluctuations inside the potential wells where the diffusion spends most of its time are carefully eliminated, the reduced dynamics is robust.
\end{abstract}

Keywords: Stochastic resonance, spectral gap, stochastic differential equation, energy balance model, potential diffusion, effective dynamics. 


\section{Background and paradigm}

The physical effect we shall discuss is related to the dynamics of an overdamped Brownian particle in a periodically changing double well potential landscape described by a potential function $U(x, t), x \in \mathbb{R}$, $t \geq 0$. To catch its essentials and at the same time to simplify the system we will work with a function $U$ switching discontinuously every half period between two antisymmetric time independent potential states $U_{1}$ and $U_{2}$. In the strip $(x, t) \in \mathbb{R} \times[0,1)$ it is defined by the formula
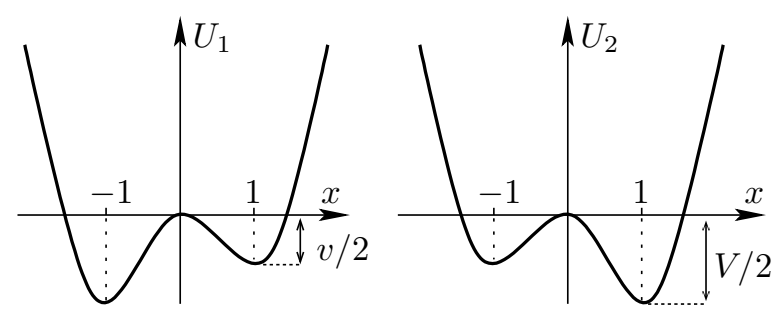

Figure 1. Time-periodic potential $U$.

$$
U(x, t)= \begin{cases}U_{1}(x), & t \in\left[0, \frac{1}{2}\right), \\ U_{2}(x)=U_{1}(-x), & t \in\left[\frac{1}{2}, 1\right) .\end{cases}
$$

It is periodically extended for all times $t$ by the relation $U(\cdot, t)=U(\cdot, t+$ $1)$, see Fig. 1. We assume that the potential has two local minima at \pm 1 and a local maximum at 0 , that $U_{1}(-1)=-\frac{V}{2}, U_{1}(1)=-\frac{v}{2}$, $\frac{2}{3}<\frac{v}{V}<1$, and $U_{1}(0)=0$. We also suppose that the extrema of $U$ are not degenerate, i.e. the curvatures at these points do not vanish.

A trajectory of a Brownian particle in this potential is described by the SDE

$$
d X_{t}^{\varepsilon, T}=-U^{\prime}\left(X_{t}^{\varepsilon, T}, \frac{t}{T}\right) d t+\sqrt{\varepsilon} d W_{t}, \quad X_{0}^{\varepsilon, T}=x \in \mathbb{R},
$$

where $\varepsilon>0$ is the noise intensity, and $T>0$ the period.

The system is said to be in stochastic resonance if the trajectories of the diffusion given by (2) amplify the small periodic forcing in the potential in a 'best possible way'. To find the stochastic resonance point, we have to determine an optimal tuning $\varepsilon=\varepsilon(T)$, i.e. to find the noise intensity for which the trajectories $X^{\varepsilon, T}$ look 'as periodic as possible'. Of course, in these terms stochastic resonance is a rather unprecise concept. To make it precise requires at least that we are able tomeasure periodicity in diffusion trajectories. 
Stochastic resonance as a tuning effect of periodically perturbed stochastic systems was first discussed in the context of a simple qualitative explanation of global glacial cycles in papers by C. Nicolis [15] and Benzi et al. $[2,3,4]$. In the context discussed there, the periodically varying potential function interprets $X$ as the globally averaged annual temperature $T$ of the earth, and describes the balance of incoming and outgoing radiative energy in the earth's atmosphere, normalized by the heat capacity factor $c$ :

$$
-\frac{\partial}{\partial T} U(T, t)=\frac{1}{c}\left[Q(t)(1-a(T(t)))-\sigma T(t)^{4}\right] .
$$

In the simplest case considered here the second term on the right hand side, the total energy flux emitted by the earth, is assumed to be given by the Stefan-Boltzmann law of a black body radiator.

The first term on the right hand side, describing the absorbed energy flux depends on two factors. The global solar function $Q(t)$ describes the flux of the solar energy which reaches the earth at time $t$. It is supposed to fluctuate with a very long period of about $10^{5}$ years and a small amplitude around an average value, and periodicity is attributed to eccentricities in the earth's orbit around the sun caused by the gravitational influence of exterior planets of the solar system. To obtain the solar radiation absorbed by the atmosphere, one has to multiply the solar function by the proportion of the radiation reaching the atmosphere which is not reflected to space, where the reflected ratio is measured by a purely temperature dependent albedo function $a(T)$. In the simple Budyko-Sellers setting (see Budyko [5] and Sellers [18]) the graph of the albedo function takes the shape of a ramp, and therefore $U$ is a continuously varying potential function with a very large period, and in general time dependent local extrema. The potential function in (1) of our outset is just a simple caricature of the one encountered in the glacial cycles' example, still retaining its essential features.

\section{Freidlin's approach}

Using large deviations theory, M. Freidlin [9] explains periodicity as a quasi-deterministic property of diffusion trajectories for very large period lengths.

For instance, let us study a Brownian particle in the time homogeneous double well potential $U_{1}$. Its motion is determined by the SDE

$$
d X_{t}^{\varepsilon}=-U_{1}^{\prime}\left(X_{t}^{\varepsilon}\right) d t+\sqrt{\varepsilon} d W_{t} .
$$

For small $\varepsilon$, this stochastic system can be considered as a deterministic dynamical system $\dot{x}=-U_{1}^{\prime}(x)$ perturbed by white noise of small 
intensity. The Freidlin-Wentzell theory of large deviations [10] allows to study asymptotic properties of (4) in the small noise limit in terms of the geometry of the potential $U_{1}$. It is intuitively clear that for $\varepsilon$ small the trajectories of (4) spend most of the time very close to the meta-stable states \pm 1 . Jumps between the wells occur. The dynamics of these very rare transitions can be investigated by means of the quasipotential. It describes the work to be done by the diffusion in order to move between points in the potential landscape. Let, for instance, $X_{0}^{\varepsilon}=-1$ and $x$ belong to the left well. Then the quasipotential $V(-1, x)$ equals $2\left(U_{1}(x)-U_{1}(-1)\right)$ and thus corresponds to twice the height of the potential barrier between -1 and $x$. If $x$ belongs to the right well and $0<x \leq 1$, then $V(-1, x)=2\left(U_{1}(0)-U_{1}(-1)\right)$. Only the way 'up' in the potential landscape contributes to the quasipotential; the way 'down' requires no work and is free. Quasipotentials are defined for rather general classes of stochastic systems by means of action functionals, for details see [10].

Let us define the first hitting time of the point $y \in \mathbb{R}$ by

$$
\tau_{y}^{\varepsilon}=\inf \left\{t \geq 0: X_{t}^{\varepsilon}=y\right\} .
$$

Then the quasipotential at $x$ and $y$ determines the exponential order of $\tau_{y}^{\varepsilon}$ if the diffusion starts in $x$ (under the law $\mathbf{P}_{x}$ ) in the limit of small noise.

Theorem 1 ('transition law') For all $\delta>0$ the following holds:

$$
\lim _{\varepsilon \downarrow 0} \mathbf{P}_{x}\left(e^{\frac{1}{\varepsilon}(V(x, y)-\delta)}<\tau_{y}^{\varepsilon}<e^{\frac{1}{\varepsilon}(V(x, y)+\delta)}\right)=1 .
$$

The most important statement of the the theorem is that the system (4) has two different intrinsic time scales of exponential order: the exit time from the left well is of the order $e^{V / \varepsilon}$ whereas the exit time from the right well of the order $e^{v / \varepsilon}$. This has the following consequence. If we consider the diffusion (4) on the exponentially long time intervals $T_{\varepsilon} \propto e^{\lambda / \varepsilon}$, then for $0<\lambda<v$ the trajectories typically cannot leave the initial well, whereas for $\lambda>v$ they spend most of their time near the deep valley (in probability). In other words, on different exponential time scales the system (4) has different meta-stable states.

For the time inhomogeneous system (2) this description of meta-stable behaviour has the following consequences. Let the period $T=T_{\varepsilon}$ be such that $\lim _{\varepsilon \downarrow 0} \varepsilon \log T_{\varepsilon}=\lambda>0$. Then for $\lambda<v$ the diffusion does not have enough energy to leave even the shallow well during one half period, and therefore, as in the time homogeneous case, is stuck in its initial well. However, if $\lambda>v$, the following effect of quasi-periodicity appears. 
Theorem 2 ([9]) Let the process $X^{\varepsilon, T}$ satisfy (2), and

$$
\lim _{\varepsilon \downarrow 0} \varepsilon \log T_{\varepsilon}>v .
$$

Then for all $A>0$ and $\delta>0$ the following holds true:

$$
\Lambda\left\{t \in[0, A]:\left|X_{T_{\varepsilon} t}^{\varepsilon, T}-\phi(t)\right|>\delta\right\} \rightarrow 0
$$

in $\mathbf{P}_{x}$-probability as $\varepsilon \rightarrow 0$, where $\Lambda\{\cdot\}$ denotes Lebesgue measure on $\mathbb{R}$, and

$$
\phi(t)= \begin{cases}-1, & t(\bmod 1) \in\left[0, \frac{1}{2}\right), \\ 1, & t(\bmod 1) \in\left[\frac{1}{2}, 1\right),\end{cases}
$$

is the coordinate of the global minimum of $U(\cdot, t)$, see Fig. 2.
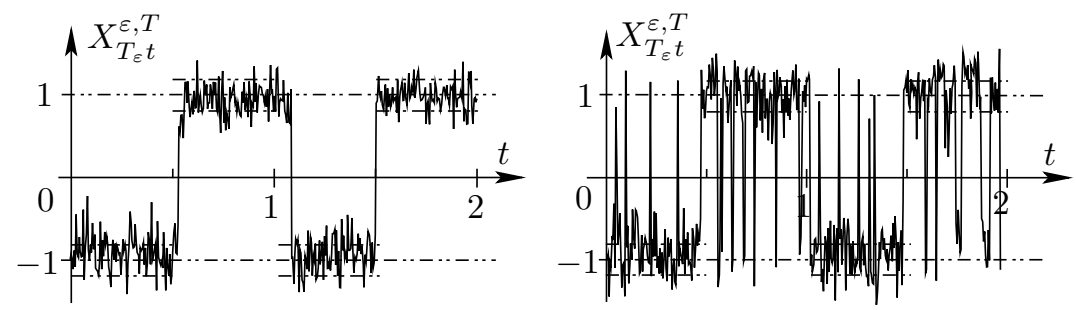

Figure 2. On time intervals satisfying condition (5) the diffusion $X^{\varepsilon, T}$ is close to the deterministic periodic function $\phi$.

Theorem 2 suggests a measure of periodicity of diffusion trajectories: the Lebesgue measure of those times the trajectories spend outside of a $\delta$-tube around the deterministic discontinuous periodic function $\phi$. Condition (5) on period $T_{\varepsilon}$ and noise intensity $\varepsilon$ provides a family of tunings. Now a resonance point in the sense explained above would require to choose $\varepsilon$ such that the above Lebesgue measure is minimal for given large period. Such an extreme value is not suggested by Freidlin's approach.

\section{Spectral power amplification}

The coefficient of spectral power amplification (SPA) is another measure of periodic tuning of random trajectories. In fact it appears to be the physicists' favorite measure, see e.g. $[4,14,11,1,6]$. For the diffusion (2) it is given by

$$
\eta^{X}(\varepsilon, T)=\left|\int_{0}^{1} \mathbf{E}_{\mu}\left(X_{T s}^{\varepsilon, T}\right) \cdot e^{2 \pi i s} d s\right|^{2} .
$$


The function $\eta^{X}$ depending on noise intensity and the period of time variation of the potential has a clear physical meaning. It describes the amount of energy carried by the average path of the diffusion with noise amplitude $\varepsilon$ on the frequency $\frac{2 \pi}{T}$. The expectation $\mathbf{E}_{\mu}$ indicates that averages are taken with respect to the invariant law of $X^{\varepsilon, T}$. This will be explained in detail later.

Fig. 3 borrowed from [1] where $\Omega$ corresponds to our $\frac{2 \pi}{T}$ and $D$ to the diffusion intensity $\varepsilon$ shows that physicists expect a local maximum of the function $\varepsilon \mapsto \eta^{X}(\varepsilon, \cdot)$. It is taken for a value of $\varepsilon$ for which the random sample paths have their strongest periodic component. In fact, Fig. 3 depicts not the SPA coefficient of the diffusion itself, but of its so-called 'effective dynamics'. The 'effective dynamics' of a diffusion in a double well potential is described by a two-state Markov chain living on the meta-stable states \pm 1 . It reflects only the interwell dynamics of the diffusion and neglects small fluctuations inside the wells. It is generally believed in the physical literature that the 'effective dynamics' adequately describes the asymptotic properties of the diffusion in the limit of small noise.

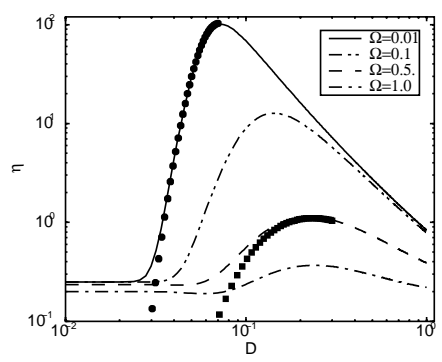

Figure 3. SPA coefficient as a function of noise amplitude has a well pronounced maximum depending at the frequency of periodic perturbation [1].

If periodic tuning is measured by SPA, the 'optimal tuning' or stochastic resonance point is determined by finding the argument $\varepsilon=\varepsilon(T)$ of a local maximum of $\varepsilon \mapsto \eta^{X}(\varepsilon, \cdot)$.

The key step to solve this problem consists in determining the density $\mu$ of the invariant law of $\left(X_{T t}^{\varepsilon, T}\right)_{t \geq 0}$. We now follow [17] and [13]. By enlarging the state space we can eliminate the inhomogeneity due to periodicity of $U$ and consider a two-dimensional time homogeneous Markov process $\left(X_{T t}^{\varepsilon, T}, t(\bmod 1)\right)$ which possesses an invariant law in the usual sense. The invariant density is a positive solution of the forward 
Kolmogorov (Fokker-Planck) equation $A_{\varepsilon, T}^{*} \mu=0$, where

$$
A_{\varepsilon, T^{*}}^{*}=-\frac{1}{T} \frac{\partial}{\partial t} \cdot+\frac{\varepsilon}{2} \frac{\partial^{2}}{\partial x^{2}} \cdot+\frac{\partial}{\partial x}\left(\cdot \frac{\partial}{\partial x} U\right)
$$

is the formal adjoint of the infinitesimal generator of the two-dimensional diffusion. From the time periodicity and time-space antisymmetry of the potential $U(1)$ the following constraints arise: $\mu(x, t)=\mu\left(-x, t+\frac{1}{2}\right)$ and $\mu(x, t)=\mu(x, t+1),(x, t) \in \mathbb{R} \times \mathbb{R}_{+}$.

These equations may be summarized in the following boundary-value problem used to determine $\mu$. It is easily seen to reduce to the FokkerPlanck equation $A_{\varepsilon, T}^{*} \mu=0$ in the strip $(x, t) \in \mathbb{R} \times\left[0, \frac{1}{2}\right]$ with boundary condition $\mu(x, 0)=\mu\left(-x, \frac{1}{2}\right), x \in \mathbb{R}$.

\section{The spectral gap}

In (1) the time dependent potential $U$ is supposed to be a step function of the time variable. In the half period region $(x, t) \in \mathbb{R} \times\left(0, \frac{1}{2}\right)$ it therefore is identical to a time independent double well potential $U_{1}$, and therefore the Fokker-Planck equation turns into a one-dimensional parabolic PDE

$$
\frac{1}{T} \frac{\partial}{\partial t} \mu(x, t)=\frac{\varepsilon}{2} \frac{\partial^{2}}{\partial x^{2}} \mu(x, t)+\frac{\partial}{\partial x}\left(\mu(x, t) \frac{\partial}{\partial x} U_{1}(x)\right) .
$$

Let $L_{\varepsilon}^{*}$ denote the second order differential operator appearing on the right hand side of (7).

To determine $\mu$ we shall expand the solution of (7) into a Fourier series with respect to the system of eigenfunctions of the operator $L_{\varepsilon}^{*}$. If $U_{1}$ is smooth and increases 'fast enough' at infinity (for example, as fast as $\left.x^{4}\right)$, the operator $L_{\varepsilon}^{*}$ is essentially self-adjoint in $\mathcal{L}^{2}\left(\mathbb{R}, e^{\frac{2 U_{1}}{\varepsilon}} d x\right)$, its spectrum is discrete and non-positive, and the corresponding eigenspaces are one-dimensional. Denoting by $\|\cdot\|$ and $\langle\cdot, \cdot\rangle$ the norm and the inner product in $\mathcal{L}^{2}\left(\mathbb{R}, e^{\frac{2 U_{1}}{\varepsilon}} d x\right)$ we consider the following formal Floquet type expansion

$$
\mu(x, t)=\sum_{k=0}^{\infty} a_{k} \frac{\Psi_{k}(x)}{\left\|\Psi_{k}\right\|} e^{-T \lambda_{k} t}, \quad(x, t) \in \mathbb{R} \times\left[0, \frac{1}{2}\right],
$$

where $\left\{-\lambda_{k}, \frac{\Psi_{k}}{\left\|\Psi_{k}\right\|}\right\}_{k \geq 0}$ is the orthonormal basis corresponding associated with the spectral decomposition of $L_{\varepsilon}^{*}$. The eigenvalues are numbered in increasing order and the Fourier coefficients $a_{k}$ are obtained from the boundary condition $\mu(x, 0)=\mu\left(-x, \frac{1}{2}\right), x \in \mathbb{R}$. 
Here is the key observation opening the route towards finding local maxima of the SPA coefficient. The terms in the sum (8) decay in time exponentially fast with rates $\lambda_{k}$, and therefore the terms corresponding to larger eigenvalues contribute less than the ones belonging to the low lying eigenvalues. This underlines their key importance. Fortunately, in the case of a double well potential there is a gap in the spectrum between $\lambda_{1}$ and $\lambda_{2}$.

Theorem 3 ('spectral gap') In the limit of small noise, the following asymptotics holds:

$$
\begin{aligned}
& \lambda_{0}=\lambda_{0}(\varepsilon)=0, \text { and } \Psi_{0}=e^{-\frac{2 U_{1}}{\varepsilon}}, \\
& \lambda_{1}=\lambda_{1}(\varepsilon)=\frac{1}{2 \pi} \sqrt{U_{1}^{\prime \prime}(1)\left|U_{1}^{\prime \prime}(0)\right|} \cdot e^{-v / \varepsilon}(1+\mathcal{O}(\varepsilon)), \\
& \lambda_{2}=\lambda_{2}(\varepsilon) \geq C>0 \text { uniformly in } \varepsilon .
\end{aligned}
$$

The result of Theorem 3 plays a crucial role in our analysis. Due to the spectral gap only the first two terms of (8) can contribute essentially As indicated by Theorem to the SPA coefficient $\eta^{X}$.

\section{Asymptotics of the SPA coefficient}

As indicated by Theorem 3 we now have to investigate the asymptotics of the first two Fourier coefficients $a_{0}$ and $a_{1}$.

\section{Theorem 4}

$$
\begin{aligned}
& a_{0}=\left\|\Psi_{0}\right\|, \\
& a_{1}=\frac{\left\|\Psi_{1}\right\|}{\left\|\Psi_{0}\right\|^{2}} \cdot \frac{\left\langle\Psi_{0}(-\cdot), \Psi_{1}\right\rangle}{\left\|\Psi_{1}\right\|^{2}-e^{-\frac{1}{2} T \lambda_{1}}\left\langle\Psi_{1}(-\cdot), \Psi_{1}\right\rangle}+r
\end{aligned}
$$

where $r$ tends to 0 in the limit of small noise and for $T \geq \exp \{(v+\delta) / \varepsilon\}$, and where $\delta$ is positive and sufficiently small.

Recalling the definition (6) of the SPA coefficient we set

$$
S^{X}(\varepsilon, T)=\int_{0}^{\frac{1}{2}} \mathbf{E}_{\mu} X_{T s}^{\varepsilon, T} \cdot e^{2 \pi i s} d s,
$$

to identify $\eta^{X}=4\left|S^{X}\right|^{2}$.

Theorem 5 Consider $T \geq \exp \{(v+\delta) / \varepsilon\}$ for $\delta$ positive and sufficiently small. Then in the small noise limit $\varepsilon \rightarrow 0$ the following asymptotic expansion holds true

$$
S^{X}=\frac{1}{\pi i} b_{0}+\frac{1}{\pi i-\frac{1}{2} \lambda_{1} T} b_{1}+r_{1}
$$


where the rest term $r_{1}$ tends to zero and the coefficients are given by

$$
\begin{aligned}
& b_{0}=\frac{\int y e^{-\frac{2 U_{1}(y)}{\varepsilon}} d y}{\int e^{-\frac{2 U_{1}(y)}{\varepsilon}} d y} \\
& b_{1}=-\frac{1+e^{-\frac{1}{2} T \lambda_{1}}}{2} \cdot \frac{\int y \Psi_{1}(y) d y}{\int e^{-\frac{2 U_{1}(y)}{\varepsilon}} d y} \cdot \frac{\left\langle\Psi_{0}(-\cdot), \Psi_{1}\right\rangle}{\left\|\Psi_{1}\right\|^{2}-e^{-\frac{1}{2} T \lambda_{1}}\left\langle\Psi_{1}(-\cdot), \Psi_{1}\right\rangle} .
\end{aligned}
$$

Finally,

$$
\eta^{X}=b_{0}^{2} \frac{4}{\pi^{2}} \frac{\left(\lambda_{1} T\right)^{2}}{4 \pi^{2}+\left(\lambda_{1} T\right)^{2}}+R,
$$

where $R$ tends to zero with $\varepsilon$.

Equipped with our knowledge of the first two Fourier coefficients let us now come to the main point of this investigation, the resonance behaviour of the SPA coefficient $\eta^{X}$. We have to find out whether it has a local maximum in $\varepsilon$. The following Lemma is obtained by application of Laplace's method of asymptotic expansions of singular integrals, see $[8,16]$, see also $[17,13]$. It contains precise asymptotic estimates of the main contributions of our Fourier expansion to $\eta^{X}$.

Lemma 6 ('Laplace's method') In the small noise limit, the following holds true:

$$
\begin{aligned}
& b_{0}=-1-\frac{1}{4} \frac{U_{1}^{(3)}(-1)}{U_{1}^{\prime \prime}(-1)^{2}} \varepsilon+\mathcal{O}\left(\varepsilon^{2}\right), \\
& b_{1}=-1+\mathcal{O}(\varepsilon)
\end{aligned}
$$

and consequently

$$
\begin{aligned}
& b_{0}^{2}=1+\frac{1}{2} \frac{U_{1}^{(3)}(-1)}{U_{1}^{\prime \prime}(-1)^{2}} \varepsilon+\mathcal{O}\left(\varepsilon^{2}\right), \\
& \left(b_{0}-b_{1}\right)^{2}=\mathcal{O}\left(\varepsilon^{2}\right) .
\end{aligned}
$$

Now we can formulate our main theorem.

Theorem 7 Let us fix $\delta$ positive and sufficiently small and $\Delta>v+\delta$. Let also $U_{1}(x)-2 U_{1}(-x)<v+V$ for all $x \in \mathbb{R}$ (the potential is not strongly asymmetric!). Then for $T \rightarrow \infty$ and $\varepsilon$ from the domain

$$
\frac{v+\delta}{\log T} \leq \varepsilon \leq \frac{\Delta}{\log T}
$$


the following asymptotic expansion for the SPA coefficient holds:

$$
\eta^{X}(\varepsilon, T)=\frac{4}{\pi^{2}}\left(1+\frac{1}{2} \frac{U_{1}^{(3)}(-1)}{U_{1}^{\prime \prime}(-1)^{2}} \varepsilon\right)+\mathcal{O}\left(\frac{1}{\log ^{2} T}\right) .
$$

This result has the following surprising consequences.

Corollary 8 For $T \rightarrow \infty$ and $\varepsilon \in\left[\frac{v+\delta}{\log T}, \frac{\Delta}{\log T}\right]$ the SPA coefficient is a decreasing function of $\varepsilon$ if $U_{1}^{(3)}(-1)<0$ and an increasing function of $\varepsilon$ if $U_{1}^{(3)}(-1)>0$.

Thus, the SPA coefficient as quality measure for tuning shows no resonance in a domain above Freidlin's threshold for quasi-deterministic periodicity (Theorem 2). This contradicts the physical intuition for the 'effective dynamics'. The reason for this surprising phenomenon can only be hidden in the intrawell behaviour of the diffusion which was neglected when passing to the reduced Markov chain. We return to this question later. Let us next study mathematically the 'effective dynamics' of the diffusion (2).

\section{The 'effective dynamics': two-state Markov chains}

The idea of approximation of diffusions in potential landscapes by appropriate finite state Markov chains in the context of stochastic resonance is due to Eckmann and Thomas [7], and C. Nicolis [15]. It was further developed by McNamara and Wiesenfeld [14]. In this section we follow $[17,13]$. The discrete time case was studied in [12].

In order to catch the main features of the interwell hoppings of the diffusion (2) we consider the time inhomogeneous Markov chain $Y^{\varepsilon, T}$ living on the diffusion's meta-stable states \pm 1 . The infinitesimal generator of $Y^{\varepsilon, T}$ is periodic in time and is given by

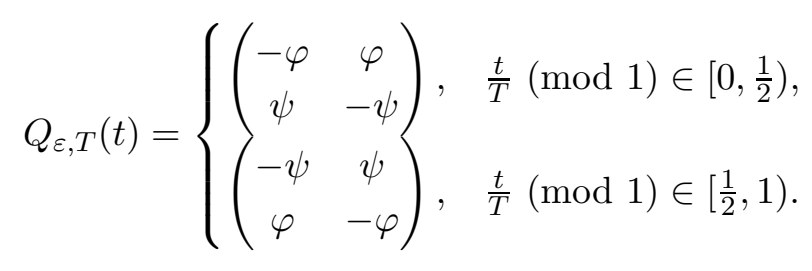

The transition rates $\varphi$ and $\psi$ have to be chosen appropriately, so that the Markov chain mimics the interwell motion of the diffusion as closely as possible. To exponential order they should correspond to the inverses of the Kramers' transition times (see Theorem 1). In their definition we 
even take care of the first sub-exponential expansion term:

$$
\varphi=\frac{1}{2 \pi} \sqrt{U_{1}^{\prime \prime}(-1)\left|U_{1}^{\prime \prime}(0)\right|} \cdot e^{-V / \varepsilon} \text { and } \psi=\frac{1}{2 \pi} \sqrt{U_{1}^{\prime \prime}(1)\left|U_{1}^{\prime \prime}(0)\right|} \cdot e^{-v / \varepsilon} .
$$

In this simplified setting, it is no surprise that quantities important for our analysis can be explicitly calculated. For instance, the invariant measure of $Y_{T t}^{\varepsilon, T}$ can be obtained as a solution of a forward Kolmogorov equation and is given by

$$
\begin{aligned}
& \nu^{-}(t)=\frac{\psi}{\varphi+\psi}+\frac{\varphi-\psi}{\varphi+\psi} \frac{e^{-(\varphi+\psi) T t}}{1+e^{-\frac{1}{2}(\varphi+\psi) T t}}, \\
& \nu^{+}(t)=\frac{\varphi}{\varphi+\psi}-\frac{\varphi-\psi}{\varphi+\psi} \frac{e^{-(\varphi+\psi) T t}}{1+e^{-\frac{1}{2}(\varphi+\psi) T t}}, \quad t \in\left[0, \frac{1}{2}\right],
\end{aligned}
$$

and $\nu^{ \pm}(t)=\nu^{\mp}\left(t+\frac{1}{2}\right)$ for $t \geq 0$.

The SPA coefficient $\eta^{Y}$ for the Markov chain $Y^{\varepsilon, T}$ is defined analogously to (6). It also can be explicitly described.

Theorem 9 For all $\varepsilon>0$ and $T>0$ the following holds:

$$
\eta^{Y}(\varepsilon, T)=\frac{4}{\pi^{2}} \frac{T^{2}(\varphi-\psi)^{2}}{4 \pi^{2}+T^{2}(\varphi+\psi)^{2}} .
$$

Compare (13) with (10). Since $(\varphi \pm \psi)^{2} \approx \lambda_{1}^{2}$ in the limit of small $\varepsilon$, the expressions for $\eta^{X}$ and $\eta^{Y}$ differ only in the 'geometric' pre-factor $b_{0}^{2}$ and the asymptotically negligible rest term $R$.

The exact formula (13) allows to give a complete account of the local maxima of $\eta^{Y}$ as a function of noise intensity for large periods $T$.

Theorem 10 In the limit $T \rightarrow \infty$ the function $\varepsilon \mapsto \eta^{Y}(\varepsilon, T)$ has a local maximum at

$$
\varepsilon(T) \approx \frac{v+V}{2} \frac{1}{\log T} .
$$

The 'resonance' behaviors of $\eta^{X}$ and $\eta^{Y}$ are quite different. Whereas the diffusion's SPA has no extremum for small $\varepsilon$, the Markov chain's always has. What can be responsible for this discrepancy? Note that the Markov chain mimics only the interwell dynamics of the diffusion. Thus, the SPA coefficient $\eta^{Y}$ measures only the spectral energy contributed by interwell jumps. On the other hand, $\eta^{X}$ also counts the numerous intrawell fluctuations of the diffusion. These fluctuations have small energy. But since the diffusion spends most of its time near \pm 1 very subtle local asymmetries of the potential at these points, expressed for 
example by the signs of the third derivative of $U_{1}$ in the wells' bottoms, become important and destroy optimal tuning.

To underpin this heuristics mathematically, let us now make the idea of neglecting the diffusion's intrawell fluctuations precise. For example, we cut off those excursions which have not enough energy to reach half the height of the potential barrier between the wells. Consider the cut-off function $g$ defined by

$$
g(x)= \begin{cases}-1, & x \in\left[x_{1}, x_{2}\right] \\ 1, & x \in\left[y_{1}, y_{2}\right] \\ x, & \text { otherwise }\end{cases}
$$

where $x_{1}<-1<x_{2}<0$ and $0<y_{1}<1<y_{2}$ are such that $U_{1}\left(x_{1}\right)=$ $U_{1}\left(x_{2}\right)=-\frac{V}{4}$ and $U_{1}\left(y_{1}\right)=U_{1}\left(y_{2}\right)=-\frac{v}{4}$, see Fig. 4. After cutting

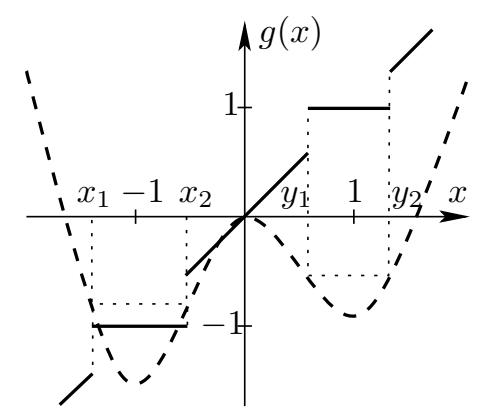

Figure 4. Function $g$ designed to cut off diffusion's intrawell dynamics.

off small fluctuations, it is clear that we have to study a modified SPA coefficient of the form

$$
\widetilde{\eta}^{X}(\varepsilon, T)=\left|\int_{0}^{1} \mathbf{E}_{\mu}\left[g\left(X_{T s}^{\varepsilon, T}\right)\right] e^{2 \pi i s} d s\right|^{2} .
$$

Following the steps of Section 5 we obtain a formula for $\widetilde{\eta}^{X}$ which is quite similar to (10) and (13):

$$
\widetilde{\eta}^{X}(\varepsilon, T)=\widetilde{b}_{0}^{2} \frac{4}{\pi^{2}} \frac{\left(\lambda_{1} T\right)^{2}}{4 \pi^{2}+\left(\lambda_{1} T\right)^{2}}+\widetilde{R}
$$

where $\widetilde{R}$ is a small rest term, and

$$
\widetilde{b}_{0}^{2}=\left(\frac{\int g(y) e^{-\frac{2 U_{1}(y)}{\varepsilon}} d y}{\int e^{-\frac{2 U_{1}(y)}{\varepsilon}} d y}\right)^{2}=1-4 \sqrt{\frac{U_{1}^{\prime \prime}(-1)}{U_{1}^{\prime \prime}(1)}} e^{-\frac{V-v}{\varepsilon}}(1+\mathcal{O}(\varepsilon))
$$


(compare to (11)).

The modified geometric pre-factor $\widetilde{b}_{0}^{2}$ is essentially smaller than its counterpart $b_{0}^{2}$. This affects the SPA coefficient $\widetilde{\eta}^{X}$ crucially: in the limit of large period and small noise its behaviour now approaches the one of the reduced Markov chain.

Theorem 11 Let the assumptions of Theorem 7 hold. Then for any $\gamma>$ 1 in the limit $T \rightarrow \infty$ the function $\varepsilon \mapsto \widetilde{\eta}^{X}(\varepsilon, T)$ has a local maximum on

$$
\left[\frac{1}{\gamma} \frac{v+V}{2} \frac{1}{\log T}, \gamma \frac{v+V}{2} \frac{1}{\log T}\right] .
$$

In other words, the optimal tuning for the measure of goodness $\widetilde{\eta}^{X}$ exists and is given approximately by

$$
\varepsilon(T) \approx \frac{v+V}{2} \frac{1}{\log T} .
$$





\section{References}

[1] V. S. Anishchenko, A. B. Neiman, F. Moss, and L. Schimansky-Geier. Stochastic resonance: noise-enhanced order. Physics-Uspekhi, 42(1):7-36, 1999.

[2] R. Benzi, G. Parisi, A. Sutera, and A. Vulpiani. The mechanism of stochastic resonance. J. Phys. A, 14:453-457, 1981.

[3] R. Benzi, G. Parisi, A. Sutera, and A. Vulpiani. Stochastic resonance in climatic changes. Tellus, 34:10-16, 1982.

[4] R. Benzi, G. Parisi, A. Sutera, and A. Vulpiani. A theory of stochastic resonance in climatic change. SIAM J. Appl. Math., 43:563-578, 1983.

[5] M. I. Budyko. The effect of solar radiation variations on the climate of the earth. Tellus, 21:611-619, 1969.

[6] M. I. Dykman, D. G. Luchinskii, R. Mannella, P. V. E. McClintock, N. D. Stein, and N. G. Stocks. Stochastic resonance in perspective. Nuovo Cimento $D, 17: 661-683,1995$.

[7] J.-P. Eckmann and L. E. Thomas. Remarks on stochastic resonance. J. Phys. A, 15:261-266, 1982.

[8] A. Erdélyi. Asymptotic expansions. Dover Publications, Inc., New York, 1956.

[9] M. I. Freidlin. Quasi-deterministic approximation, metastability and stochastic resonance. Physica D, 137(3-4):333-352, 2000.

[10] M. I. Freidlin and A. D. Wentzell. Random perturbations of dynamical systems, volume 260 of Grundlehren der Mathematischen Wissenschaften. Springer, New York, NY, second edition, 1998.

[11] L. Gammaitoni, P. Hänggi, P. Jung, and F. Marchesoni. Stochastic resonance. Reviews of Modern Physics, 70:223-287, January 1998.

[12] P. Imkeller and I. Pavlyukevich. Stochastic resonance in two-state Markov chains. Arch. Math., 77(1):107-115, 2001.

[13] P. Imkeller and I. Pavlyukevich. Model reduction and stochastic resonance. Stochastics and Dynamics, 4, 2002.

[14] B. McNamara and K. Wiesenfeld. Theory of stochastic resonance. Physical Review A (General Physics), 39:4854-4869, May 1989. 
[15] C. Nicolis. Stochastic aspects of climatic transitions - responses to periodic forcing. Tellus, 34:1-9, 1982.

[16] F. W. J. Olver. Asymptotics and special functions. Computer Science and Applied Mathematics. Academic Press, a subsidiary of Harcourt Brace Jovanovich, Publishers, New York - London, 1974.

[17] I. E. Pavlyukevich. Stochastic Resonance. PhD thesis, Humboldt-Universität, Berlin, 2002. Logos-Verlag, ISBN 3-89722-960-9.

[18] W. B. Sellers. A global climate model based on the energy balance of the earthatmosphere system. J. Appl. Meteor., 8:301-320, 1969. 